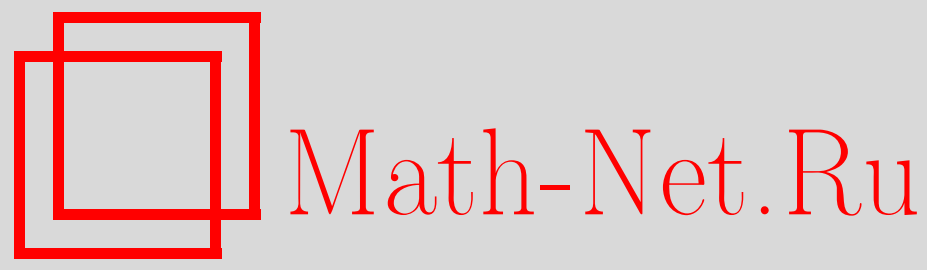

F. Götze, A. Yu. Zaitsev, Bounds for the Rate of Strong Approximation in the Multidimensional Invariance Principle, Теория вероятн. и ее примен., 2008, том 53, выпуск 1, 100-123

DOI: https://doi.org/10.4213/tvp2484

Использование Общероссийского математического портала MathNet.Ru подразумевает, что вы прочитали и согласны с пользовательским соглашением

http: //www.mathnet.ru/rus/agreement

Параметры загрузки:

IP : 3.85 .7 .115

26 апреля 2023 г., $16: 45: 23$

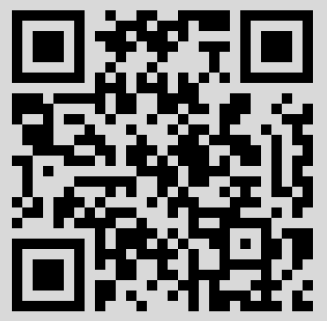




\title{
BOUNDS FOR THE RATE OF STRONG APPROXIMATION IN THE MULTIDIMENSIONAL INVARIANCE PRINCIPLE ${ }^{1)}$
}

\begin{abstract}
Цель работы - доказать некоторые следствия работы второго автора [34]. Мы устанавливаем оценки для скорости сильной гауссовской аппроксимации сумм независимых $\mathbf{R}^{d}$-значных случайных векторов $\xi_{j}$, имеющих конечные моменты $\mathbf{E}\left\|\xi_{j}\right\|^{\gamma}, \gamma \geqslant 2$. Получены многомерные аналоги результатов работы А.И. Саханенко [27].

Ключевые слова и фразы: многомерный принцип инвариантности, сильная аппроксимация, суммы независимых случайных векторов.
\end{abstract}

1. Introduction. The aim of this paper is to derive consequences of a result of [34] providing optimal bounds for the strong Gaussian approximation of sums of independent $\mathbf{R}^{d}$-valued random vectors with finite exponential moments (see Theorem 3 below). Theorem 3 may be considered as a multidimensional generalization of well-known results of Komlós, Major, and Tusnády (KMT) [19] and Sakhanenko [26], who generalized and essentially sharpened KMT results in the case of nonidentically distributed $\mathbf{R}^{1}$-valued random variables. Then, Sakhanenko [27] used the main result of [26] to obtain optimal bounds for the strong Gaussian approximation of sums of independent random variables $\xi_{j}$ having finite moments of the form $\mathbf{E} H\left(\left|\xi_{j}\right|\right)$, where $H(x)$ is a monotone function growing not slower than $x^{2}$ and not faster than $e^{c x}$. The most fine and complete results were obtained in the case $H(x)=x^{\gamma}, \gamma \geqslant 2$. In this paper, basing on the results of [34], we provide a new attempt to obtain a multidimensional generalization of the results of [27], restricting ourselves to the case $H(x)=x^{\gamma}$. The first attempt was realized in [36] (see Theorem 2 below), but the corresponding inequality was established not for all possible values of the argument $z$.

* Fakultät für Mathematik, Universität Bielefeld, Postfach 100131, 33501 Bielefeld 1, Germany; e-mail: goetze@math.uni-bielefeld.de

** С.-Петербургское отделение Математического института им. В. А. Стеклова PAН, ул. Фонтанка, 27, 191023 C.-Петербург, Россия; e-mail: zaitsev@pdmi.ras.ru

1) Research was supported by the SFB 701, University of Bielefeld; research of the second author was supported by Russian Foundation of Basic Research (RFBR) Grant 0501-00911, by RFBR-DFG Grant 04-01-04000, by the Grant of leading scientific schools NSh 4222.2006 .1 and by a program of fundamental researches of the RAS «Modern problems of fundamental mathematics». 
The problem of strong approximation of sums of independent random vectors is more delicate than that of estimating the closeness of distributions. It is required to construct on a probability space a sequence of independent random vectors $X_{1}, \ldots, X_{n}$ (with given distributions) and a corresponding sequence of independent Gaussian random vectors $Y_{1}, \ldots, Y_{n}$ so that the quantity

$$
\Delta(X, Y)=\max _{1 \leqslant k \leqslant n}\left\|\sum_{i=1}^{k} X_{i}-\sum_{i=1}^{k} Y_{i}\right\|
$$

would be so small as possible with sufficiently large probability. The estimation of the rate of strong approximation in the invariance principle may be reduced to this problem.

The rate of strong approximation in the one-dimensional invariance principle was studied by many authors (see, for example, [23], [31], [3], [6], [19] and the bibliography in the monograph [7] and in the papers [5], $[8],[20],[30])$. One should mention the recent results of Sakhanenko [29], who sharpened the inequalities of [27] expressing the bounds in terms of truncated moments.

Multidimensional estimates in the strong invariance principle can be found in [17], [2], [22], [4], [1], [12], [13], [28]. The most of these papers contain the results for sufficiently small values of $\gamma \geqslant 2$ or bounds for the Prokhorov distance and for similar quantities. The Prokhorov distance estimation (coupled with the well-known Strassen-Dudley theorem) allows one to get the statements about the strong approximation for concrete values of the argument $z$ (see (1.3), (1.7), and (1.16)-(1.18)). This means that the probability space involved in the results depends on this $z$.

Einmahl [14], obtained multidimensional results about the optimal rate of the strong Gaussian approximation of infinite sequences of sums of i.i.d. random vectors with $\mathbf{E} H\left(\left\|\xi_{1}\right\|\right)<\infty$. In particular, he established the optimal rate in the case $H(x)=x^{\gamma}, \gamma>3$. Similarly to [19] and [27], the proof in [14], was based on the result for the vectors with finite exponential moments. The latter contained an extra logarithmic factor in the corresponding inequalities. Zaitsev [33], [34] removed this unnecessary logarithmic factor from the result of [14] and obtained multidimensional analogues of the KMT and Sakhanenko results for the strong Gaussian approximation of sums of independent $\mathbf{R}^{d}$-valued random vectors with finite exponential moments.

Below we need some notation. The distribution of a random vector $\xi$ will be denoted by $\mathscr{L}(\xi)$. The corresponding covariance operator will be denoted by $\operatorname{cov} \xi$ or $\operatorname{cov} V$, if $V=\mathscr{L}(\xi)$. We denote by $c$ universal constants which might be different in different places of the text. We write $\ln ^{*} b=$ $\max \{1, \ln b\}$, for $b>0$.

The aim of the present paper is to obtain multidimensional analogues of the following result of Sakhanenko. 
Theorem 1 ([27]). Let $\xi_{1}, \ldots, \xi_{n}$ be independent random variables with $\mathbf{E} \xi_{j}=0, j=1, \ldots, n$. Let $\gamma \geqslant 2$ and

$$
L_{\gamma}=\sum_{j=1}^{n} \mathbf{E}\left|\xi_{j}\right|^{\gamma}<\infty .
$$

Then one can construct on a probability space a sequence of independent random variables $X_{1}, \ldots, X_{n}$ and a corresponding sequence of independent Gaussian random variables $Y_{1}, \ldots, Y_{n}$ so that $\mathscr{L}\left(X_{j}\right)=\mathscr{L}\left(\xi_{j}\right), \mathbf{E} Y_{j}=0$, $\mathrm{D} Y_{j}=\mathbf{D} X_{j}, j=1, \ldots, n$,

$$
\mathbf{E}(\Delta(X, Y))^{\gamma} \leqslant c \gamma^{2 \gamma} L_{\gamma}
$$

and, hence, for all $z>0$,

$$
\mathbf{P}\{\Delta(X, Y) \geqslant z\} \leqslant c \gamma^{2 \gamma} L_{\gamma} z^{-\gamma},
$$

where $c$ is a universal constant. Moreover, there exists an absolute positive constant $C$ such that

$$
\mathbf{P}\{\Delta(X, Y) \geqslant C z\} \leqslant c \gamma^{2 \gamma b} b^{\gamma b}\left(L_{\gamma} z^{-\gamma}\right)^{b}+2 \sum_{j=1}^{n} \mathbf{P}\left\{\left\|\xi_{j}\right\| \geqslant \frac{z}{b}\right\},
$$

for any $b \geqslant 1$ and $z \geqslant 0$.

It should be mentioned that, in [27], one can find more general results. In particular, there was considered the case, where the random variable $\xi_{j}$ have finite moments of the form $\mathbf{E} H\left(\left|\xi_{j}\right|\right)$, where $H(x)$ is a monotone function growing not slower than $x^{2}$ and not faster than $e^{c x}$. The results of [27] are very precise and general. They imply a lot of other approximation results (see [30]). In particular, in [27] it is observed that inequality (1.2) implies the well-known Rosenthal [24], [25] inequality (see Lemma 3 below).

After the natural normalization, we see that (1.2) is equivalent to

$$
\mathbf{E}\left(\Delta(X, Y) \sigma^{-1}\right)^{\gamma} \leqslant c \gamma^{2 \gamma} L_{\gamma} \sigma^{-\gamma}
$$

where $\sigma^{2}=\mathbf{D}\left(\sum_{j=1}^{n} \xi_{j}\right)$. It is clear that $L_{\gamma} / \sigma^{\gamma}, 2<\gamma \leqslant 3$, is the wellknown Lyapunov fraction involved in the Lyapunov and Esséen bounds for the Kolmogorov distance in the central limit theorem.

The following Theorem 2 was proved in a recent publication of Zaitsev (see $[36])$.

Theorem 2 ([36]). Suppose that $\alpha>0$ and $\xi_{1}, \ldots, \xi_{n}$ are independent $\mathbf{R}^{d}$-valued random vectors with $\mathbf{E} \xi_{j}=0, j=1, \ldots, n$. Let $\gamma \geqslant 2$ and

$$
L_{\gamma}=\sum_{j=1}^{n} \mathbf{E}\left\|\xi_{j}\right\|^{\gamma}<\infty
$$

Assume that there exist a positive integer $s$ and a strictly increasing sequence of nonnegative integers $m_{0}=0, m_{1}, \ldots, m_{s}=n$ satisfying the following conditions. Let

$$
\zeta_{k}=\xi_{m_{k-1}+1}+\cdots+\xi_{m_{k}}, \quad \operatorname{cov} \zeta_{k}=\mathbb{B}_{k}, \quad k=1, \ldots, s,
$$


and assume that, for all $v \in \mathbf{R}^{d}$ and $k=1, \ldots, s$,

$$
r^{2} L_{\gamma}^{2 / \gamma}\|v\|^{2} \leqslant\left\langle\mathbb{B}_{k} v, v\right\rangle \leqslant C r^{2} L_{\gamma}^{2 / \gamma}\|v\|^{2}
$$

with some constants $r \geqslant 2 e$ and $C \geqslant 1$. Then one can construct on $a$ probability space a sequence of independent random vectors $X_{1}, \ldots, X_{n}$ and a corresponding sequence of independent Gaussian random vectors $Y_{1}, \ldots, Y_{n}$ so that $\mathscr{L}\left(X_{j}\right)=\mathscr{L}\left(\xi_{j}\right), \mathbf{E} Y_{j}=0, \operatorname{cov} Y_{j}=\operatorname{cov} X_{j}, j=1, \ldots, n$, and, for $z>a_{1} d^{15 / 2+\alpha} \ln ^{*} d \cdot r L_{\gamma}^{1 / \gamma} \ln ^{*} s$

$$
\mathbf{P}\{\Delta(X, Y) \geqslant 5 z\} \leqslant 2 L_{\gamma} z^{-\gamma}+\exp \left(-\frac{a_{2} z}{r L_{\gamma}^{1 / \gamma} d^{9 / 2} \ln ^{*} d}\right),
$$

where $a_{1}, a_{2}$ are positive quantities depending only on $\alpha$ and $C$.

Theorem 2 provides the multidimensional analogue of a weakened version of inequality (1.3). Roughly speaking, in inequality (1.3) of Theorem 1 the statement is the same as in Theorem 2 but without the exponential term in (1.7), without condition (1.6) and without any restrictions on $z>0$. If $\mathbf{D}\left(\sum_{j=1}^{n} \xi_{j}\right) \geqslant 32 L_{\gamma}^{2 / \gamma}$, then condition (1.6) is always satisfied in the onedimensional situation since $\mathbf{D} \xi_{j} \leqslant\left(\mathbf{E}\left|\xi_{j}\right|^{\gamma}\right)^{2 / \gamma} \leqslant L_{\gamma}^{2 / \gamma}, j=1, \ldots, n$, and we can find $m_{1}, \ldots, m_{s}$ so that (1.6) is valid with $e^{-1} r=C=2$. Note that, for $z \leqslant L_{\gamma}^{1 / \gamma}$, inequality (1.7) is satisfied trivially. It is evident that, in the case $d=1, e^{-1} r=C=2$, the exponential term may easily be estimated by $c \gamma^{\gamma} L_{\gamma} z^{-\gamma}$, for $z>L_{\gamma}^{1 / \gamma}$, with an absolute constant $c$. In the degenerate case, where $\mathbf{D}\left(\sum_{j=1}^{n} \xi_{j}\right) \leqslant 32 L_{\gamma}^{2 / \gamma}$, the required bounds are valid for any constructing the random vectors $\left\{X_{j}\right\}$ and $\left\{Y_{j}\right\}$ on the same probability space (see Proposition 1 below).

Thus, the only difference with Sakhanenko's inequality (1.3) is in the logarithmic factor $\ln ^{*} s$ in the restriction on $z$.

It is clear that $\ln ^{*} s$ behaves as $\ln ^{*} n$ in the case of i.i.d. $\xi_{1}, \ldots, \xi_{n}$ and as the logarithm of variances of coordinates of sums $\xi_{1}+\cdots+\xi_{n}$ in the general case. Therefore, it is natural to try to establish a multidimensional analogue of inequality (1.3) which is valid without any restrictions on $z$ as well as those of inequalities (1.2) and (1.4). Under some additional conditions, this is realized in Theorem 4 below, which provides a partial solution of the problem.

Note that one can find in [36] the corresponding versions of inequality (1.7) for the vectors with finite moments of the form $\mathbf{E} H\left(\left\|\xi_{j}\right\|\right)$, where $H(x)$ is a monotone function growing not slower than $x^{2}$ and not faster than $e^{c x}$. Unfortunately, in these results there are similar logarithmic factors $\ln ^{*} s$ in the restrictions on $z$. Such functions were also considered in [27].

The main tool for the proof of Theorems 2 and 4 is the following Theorem 3. It was proved in [34]. In this theorem, the most important case, 
where the summands have finite exponential moments, was considered. The main result of [26] was successively generalized to the multidimensional case.

Let $\mathscr{A}_{d}(\tau), \tau \geqslant 0, d \in \mathbf{N}$, denote classes of $d$-dimensional distributions, introduced in [32], see as well [33]-[35]. The class $\mathscr{A}_{d}(\tau)$ (with a fixed $\tau \geqslant 0$ ) consists of $d$-dimensional distributions $V$ for which the function

$$
\varphi(z)=\varphi(V, z)=\ln \int_{\mathbf{R}^{d}} e^{\langle z, x\rangle} V\{d x\} \quad(\varphi(0)=0)
$$

is defined and analytic for $\|z\| \tau<1, z \in \mathbf{C}^{d}$, and

$$
\left|d_{u} d_{v}^{2} \varphi(z)\right| \leqslant\|u\| \tau\langle\mathbb{D} v, v\rangle,
$$

for all $u, v \in \mathbf{R}^{d}$ and $\|z\| \tau<1$, where $\mathbb{D}=\operatorname{cov} V$ and $d_{u} \varphi$ is the derivative of the function $\varphi$ in direction $u$. Below we shall use simplest properties of the classes $\mathscr{A}_{d}(\tau)$ which are listed in [35].

Theorem 3 ([34]). Suppose that $\alpha>0, \tau \geqslant 1$, and $\xi_{1}, \ldots, \xi_{n}$ are independent $\mathbf{R}^{d}$-valued random vectors with $\mathbf{E} \xi_{j}=0, j=1, \ldots, n$. Assume that there exist a positive integer $s$ and a strictly increasing sequence of nonnegative integers $m_{0}=0, m_{1}, \ldots, m_{s-1}, m_{s}=n$ satisfying the following conditions. Let

$\zeta_{k}=\xi_{m_{k-1}+1}+\cdots+\xi_{m_{k}}, \quad \operatorname{cov} \zeta_{k}=\mathbb{B}_{k}, \quad \mathscr{L}\left(\zeta_{k}\right) \in \mathscr{A}_{d}(\tau), \quad k=1, \ldots, s$, and suppose that, for all $v \in \mathbf{R}^{d}$,

$$
C_{1}\|v\|^{2} \leqslant\left\langle\mathbb{B}_{k} v, v\right\rangle \leqslant C_{2}\|v\|^{2}, \quad k=1, \ldots, s,
$$

with some positive constants $C_{1}$ and $C_{2}$. Then one can construct on a probability space a sequence of independent random vectors $X_{1}, \ldots, X_{n}$ and a corresponding sequence of independent Gaussian random vectors $Y_{1}, \ldots, Y_{n}$ so that $\mathscr{L}\left(X_{j}\right)=\mathscr{L}\left(\xi_{j}\right), \mathbf{E} Y_{j}=0, \operatorname{cov} Y_{j}=\operatorname{cov} X_{j}, j=1, \ldots, n$, and

$$
\mathbf{E} \exp \left(\frac{a_{3} \Delta(X, Y)}{\tau d^{9 / 2} \ln ^{*} d}\right) \leqslant \exp \left(a_{4} d^{3+\alpha} \ln ^{*} \frac{s}{\tau^{2}}\right),
$$

where $a_{3}, a_{4}$ are positive quantities depending on $\alpha, C_{1}, C_{2}$ only.

The i.i.d. version of Theorem 3 was proved by Zaitsev in [33], where he removed an additional logarithmic factor from a result of [14] and obtained a multidimensional analogue of the KMT result. Theorem 3 provides an adequate multidimensional generalization of the main result of [26]. In Theorem 3, the random vectors are, generally speaking, nonidentically distributed. However, they have to satisfy condition (1.10) on the covariance operators. Condition (1.10) generates conditions (1.6) and (1.12). A plausible conjecture is that condition (1.10) could be weakened in the statement of Theorem 3. The same may be said about conditions (1.6) and (1.12) in our results. At least, no similar restrictions are imposed in [32] (which, however, is not related to the invariance principle). But the proof of this conjecture would require some new ideas and methods.

The main result of this paper is the following theorem. 
Theorem 4. Suppose that $\alpha>0$ and $\xi_{1}, \ldots, \xi_{n}$ are independent $\mathbf{R}^{d_{-}}$ valued random vectors with $\mathbf{E} \xi_{j}=0, j=1, \ldots, n$. Let $\gamma \geqslant 2$ and let the quantity $L_{\gamma}$ be defined by (1.5). Assume that there exist a positive integer $s$ and a strictly increasing sequence of nonnegative integers $m_{0}=0$, $m_{1}, \ldots, m_{s-1}, m_{s}=n$ satisfying the following conditions. Let

$$
\zeta_{k}=\xi_{m_{k-1}+1}+\cdots+\xi_{m_{k}}, \quad \operatorname{cov} \zeta_{k}=\mathbb{B}_{k}, \quad k=1, \ldots, s,
$$

and assume that, for all $v \in \mathbf{R}^{d}$ and $k=1, \ldots, s$,

$$
w^{2}\|v\|^{2} \leqslant\left\langle\mathbb{B}_{k} v, v\right\rangle \leqslant C_{1} w^{2}\|v\|^{2}
$$

where

$$
w=C_{2} \frac{L_{\gamma}^{1 / \gamma}}{\ln ^{*} s}
$$

with some positive constants $C_{1}$ and $C_{2}$. Suppose that the quantities

$$
\lambda_{k, \gamma}=\sum_{j=m_{k-1}+1}^{m_{k}} \mathbf{E}\left\|\xi_{j}\right\|^{\gamma}, \quad k=1, \ldots, s,
$$

satisfy, for some $0<\varepsilon<1$,

$$
C_{3} d^{\gamma / 2} s^{\varepsilon}\left(\ln ^{*} s\right)^{\gamma+3} \max _{1 \leqslant k \leqslant s} \lambda_{k, \gamma} \leqslant L_{\gamma}
$$

with a positive constant $C_{3}$. Then one can construct on a probability space a sequence of independent random vectors $X_{1}, \ldots, X_{n}$ and a corresponding sequence of independent Gaussian random vectors $Y_{1}, \ldots, Y_{n}$ so that $\mathscr{L}\left(X_{j}\right)=\mathscr{L}\left(\xi_{j}\right), \mathbf{E} Y_{j}=0, \operatorname{cov} Y_{j}=\operatorname{cov} X_{j}, j=1, \ldots, n$, and, for any $z>a_{5} \varepsilon^{-1} L_{\gamma}^{1 / \gamma} d^{21 / 2+\alpha} \ln ^{*} d$ and any $b \geqslant 1$,

$$
\begin{aligned}
\mathbf{P}\{\Delta(X, Y) \geqslant 9 z\} \leqslant & 10 d^{1+\gamma b / 2}(2 b)^{b(\gamma-1)} z^{-\gamma b} \sum_{k=1}^{s} \lambda_{k, \gamma}^{b}+\exp \left(-\frac{a_{6} z^{2}}{d L_{\gamma}^{2 / \gamma}}\right) \\
& +\exp \left(-\frac{a_{7} \varepsilon z \ln ^{*} s}{L_{\gamma}^{1 / \gamma} d^{15 / 2} \ln ^{*} d}\right) \\
& +5 d \sum_{j=1}^{n} \mathbf{P}\left\{\left\|\xi_{j}\right\| \geqslant \frac{z}{2 b \sqrt{d}}\right\} \\
\mathbf{P}\{\Delta(X, Y) \geqslant 9 z\} \leqslant & d^{1+\gamma / 2}\left(a_{8} b^{4} \varepsilon^{-1}\right)^{\gamma b}\left(L_{\gamma} z^{-\gamma}\right)^{b} \\
& +5 d \sum_{j=1}^{n} \mathbf{P}\left\{\left\|\xi_{j}\right\| \geqslant \frac{z}{2 b \sqrt{d}}\right\} \\
\mathbf{P}\{\Delta(X, Y) \geqslant z\} \leqslant & a_{9} d^{1+\gamma / 2} L_{\gamma}(\varepsilon z)^{-\gamma}
\end{aligned}
$$

and

$$
\mathbf{E}(\Delta(X, Y))^{\gamma} \leqslant a_{10}\left(\varepsilon^{-1} d^{21 / 2+\alpha} \ln ^{*} d\right)^{\gamma} L_{\gamma}
$$

where $a_{5}, \ldots, a_{10}$ are positive quantities depending on $\alpha, \gamma, C_{1}, C_{2}$, and $C_{3}$ only. 
Note that, for $z \leqslant a_{5} \varepsilon^{-1} L_{\gamma}^{1 / \gamma} d^{21 / 2+\alpha} \ln ^{*} d$, we have, under conditions of Theorem 4,

$$
\mathbf{P}\{\Delta(X, Y) \geqslant z\} \leqslant 1 \leqslant\left(a_{5} \varepsilon^{-1} d^{21 / 2+\alpha} \ln ^{*} d\right)^{\gamma} L_{\gamma} z^{-\gamma} .
$$

On the other hand, inequality (1.19) implies that

$$
\mathbf{P}\{\Delta(X, Y) \geqslant z\} \leqslant a_{10}\left(\varepsilon^{-1} d^{21 / 2+\alpha} \ln ^{*} d\right)^{\gamma} L_{\gamma} z^{-\gamma},
$$

for all $z>0$. Thus, in Theorem 4, the inequality of type (1.3) is established, in fact, for all possible values of $z$. It is impossible, however, to derive from (1.19) inequality (1.18) which is sharper with respect to the dependence of constants on the dimension $d$. Note that in this paper we do not try to optimize the dependence of constants on the dimension. It is important here that it is a power-type dependence. It is also clear that (1.19) does not follows from (1.20) or from (1.18). Moreover, inequality (1.17) is sometimes stronger than (1.18) (for example, if the last sum in (1.17) is equal to zero). Inequality (1.16) is sharper than (1.17) and (1.18). On the other hand, (1.17) and (1.18) are formulated simpler than (1.16).

Condition (1.15) is rather cumbersome, but it is trivially satisfied if $\xi_{1}, \ldots, \xi_{n}$ are i.i.d. random vectors and $n$ is sufficiently large. Indeed, it is clear that in this case $m_{k} \asymp k n^{1 / \gamma} / \ln ^{*} n, \lambda_{k, \gamma} \asymp n^{1 / \gamma} / \ln ^{*} n, k=1, \ldots, s$, $s \asymp n^{1-1 / \gamma} \ln ^{*} n$, and condition (1.15) turns into

$$
C_{3} d^{\gamma / 2} n^{\varepsilon(1-1 / \gamma)}\left(\ln ^{*} n\right)^{\gamma+2+\varepsilon} \leqslant A n^{1-1 / \gamma}
$$

with some $A$ which does not depend on $n$. Moreover, in the general case

$$
L_{\gamma}=\sum_{k=1}^{s} \lambda_{k, \gamma}
$$

and (1.15) has to be satisfied if the quantities $\lambda_{k, \gamma}$ have a regular behavior.

Choosing, for example, $C_{3}=d^{-\gamma / 2}$, we could remove the dependence on $d$ in condition (1.15). But, of course, this would imply that $a_{5}, \ldots, a_{10}$ depend on $d$ too.

Let $2 \leqslant \beta<\gamma$. Then, under conditions of Theorem 4 ,

$\left(\mathbf{E}(\Delta(X, Y))^{\beta}\right)^{1 / \beta} \leqslant\left(\mathbf{E}(\Delta(X, Y))^{\gamma}\right)^{1 / \gamma} \leqslant a_{10}^{1 / \gamma} \varepsilon^{-1} d^{21 / 2+\alpha} \ln ^{*} d \cdot L_{\gamma}^{1 / \gamma}$.

This bound could be better than the bound which could be obtained if we apply Theorem 4 with $\gamma=\beta$. For example, in the i.i.d. case, $L_{\gamma}^{1 / \gamma}=$ $\left(n \mathbf{E}\left\|\xi_{1}\right\|^{\gamma}\right)^{1 / \gamma} \asymp n^{1 / \gamma}$, while $L_{\beta}^{1 / \beta}=\left(n \mathbf{E}\left\|\xi_{1}\right\|^{\beta}\right)^{1 / \beta} \asymp n^{1 / \beta}$. In the general case, for a fixed $\beta$, one should try to minimize $L_{\gamma}^{1 / \gamma}, \gamma \geqslant \beta$. Note, however, that conditions (1.12) and (1.15) are different for different $\gamma$.

Conditions (1.6) and (1.12) imply that the minimal eigenvalue of $\operatorname{cov}\left(\sum_{j=1}^{n} \xi_{j}\right)$ is bounded from below by $r^{2} L_{\gamma}^{2 / \gamma}$ and $w^{2}$ respectively. Nevertheless, if the eigenvalues of $\operatorname{cov}\left(\sum_{j=1}^{n} \xi_{j}\right)$ are small, less than $C_{4}^{2} L_{\gamma}^{2 / \gamma}$, the required inequalities are valid for any constructing the random vectors $\left\{X_{j}\right\}$ and $\left\{Y_{j}\right\}$ on the same probability space. 
Proposition 1. Suppose that $\xi_{1}, \ldots, \xi_{n}$ are independent $\mathbf{R}^{d}$-valued random vectors with $\mathbf{E} \xi_{j}=0, j=1, \ldots, n$. Let $\gamma \geqslant 2$ and let the quantity $L_{\gamma}$ be defined by (1.5). Let $\sigma^{2}$ be the maximal eigenvalue of $\operatorname{cov}\left(\sum_{j=1}^{n} \xi_{j}\right)$. Assume that

$$
\sigma \leqslant C_{4} L_{\gamma}^{1 / \gamma}
$$

with some positive constant $C_{4}$. Then, for any construction on a probability space of a sequence of independent random vectors $X_{1}, \ldots, X_{n}$ and a corresponding sequence of independent Gaussian random vectors $Y_{1}, \ldots, Y_{n}$ such that $\mathscr{L}\left(X_{j}\right)=\mathscr{L}\left(\xi_{j}\right), \mathrm{E} Y_{j}=0, \operatorname{cov} Y_{j}=X_{j}, j=1, \ldots, n$, the following inequalities are valid:

$$
\begin{aligned}
\mathbf{P}\{\Delta(X, Y) \geqslant 4 z\} \leqslant & d^{1+\gamma / 2}\left(a_{11} b\right)^{b(\gamma-1)}\left(L_{\gamma} z^{-\gamma}\right)^{b} \\
& +3 d \sum_{j=1}^{n} \mathbf{P}\left\{\left\|\xi_{j}\right\| \geqslant \frac{z}{2 b \sqrt{d}}\right\}
\end{aligned}
$$

for all $z>0, b \geqslant 1$,

$$
\mathbf{E}(\Delta(X, Y))^{\gamma} \leqslant a_{12} d^{1+\gamma / 2} L_{\gamma}
$$

and, hence,

$$
\mathbf{P}\{\Delta(X, Y) \geqslant z\} \leqslant a_{12} d^{1+\gamma / 2} L_{\gamma} z^{-\gamma}
$$

where $a_{11}$ and $a_{12}$ are positive quantities depending on $\gamma$ and $C_{4}$ only.

The proof of Proposition 1 is based, in fact, on the trivial inequality $\Delta(X, Y) \leqslant \Delta(X, 0)+\Delta(0, Y)$. It remains to estimate $\Delta(X, 0)$ and $\Delta(0, Y)$.

The proof of Theorem 4 is a modified version of the corresponding proofs from [27] and [36]. The main difference is that we truncate not the initial summands $\xi_{j}$ but the sums $\zeta_{k}$ over blocks of summands $\xi_{j}$ and then show that the distributions of truncated vectors belong to the class $\mathscr{A}_{d}(\tau)$ with $\tau$ which is essentially less than the level of truncation $u$. A similar idea was used in [14]. The complete proof in [27] is yet not generalized due to technical difficulties occurring in the multidimensional case.

Below we shall need the following Lemmas 1-3.

Lemma 1. Let $X_{1}, \ldots, X_{n}$ be independent random vectors and $S_{k}=$ $X_{1}+\cdots+X_{k}, k=1, \ldots, n$. Then

$$
\mathbf{P}\left\{\max _{1 \leqslant k \leqslant n}\left\|S_{k}\right\| \geqslant 3 t\right\} \leqslant 3 \max _{1 \leqslant k \leqslant n} \mathbf{P}\left\{\left\|S_{k}\right\| \geqslant t\right\}, \quad t \geqslant 0 .
$$

Lemma 1 is a version of the Ottaviani inequality, see [10, Proposition 1.1.2]. In the form (1.26), this inequality can be found in [18] and (with 4 instead of 3 (twice)) in [15].

Lemma 2. Let $\gamma \geqslant 2, b \geqslant 1$, let $S_{n}=\psi_{1}+\cdots+\psi_{n}$ be the sum of independent random variables $\psi_{j} \in \mathbf{R}^{1}$ with $\mathbf{E} \psi_{j}=0, \mathbf{E}\left|\psi_{j}\right|^{\gamma}<\infty$, 
$j=1, \ldots, n$, and let $\mathbf{D} S_{n}=\sigma^{2}$. Then

$$
\begin{aligned}
\mathbf{P}\left\{\left|S_{n}\right| \geqslant x\right\} \leqslant & 2 \exp \left(-\frac{a_{13} x^{2}}{\sigma^{2}}\right)+2(2 b)^{b(\gamma-1)}\left(\sum_{j=1}^{n} \frac{\mathbf{E}\left|\psi_{j}\right|^{\gamma}}{x^{\gamma}}\right)^{b} \\
& +\sum_{j=1}^{n} \mathbf{P}\left\{\left|\psi_{j}\right| \geqslant \frac{x}{2 b}\right\},
\end{aligned}
$$

for all $x>0$, where $a_{13}$ is a positive quantity depending on $\gamma$ only.

Lemma 2 follows easily from the main result of [16], see also [21, Corollary 1.7].

It is easy to see that, by the Chebyshev inequality, (1.27) with $b=1$ implies that

$$
\mathbf{P}\left\{\left|S_{n}\right| \geqslant x\right\} \leqslant 2 \exp \left(-\frac{a_{13} x^{2}}{\sigma^{2}}\right)+2^{\gamma+1} \sum_{j=1}^{n} \frac{\mathbf{E}\left|\psi_{j}\right|^{\gamma}}{x^{\gamma}},
$$

for all $x>0$, under conditions of Lemma 2 .

Lemma 3. Let $\xi_{1}, \ldots, \xi_{n}$ denote independent random vectors which have mean zero and assume values in $\mathbf{R}^{d}$. Then

$$
\mathbf{E}\left\|\sum_{j=1}^{n} \xi_{j}\right\|^{\gamma} \leqslant a_{14}\left(\sum_{j=1}^{n} \mathbf{E}\left\|\xi_{j}\right\|^{\gamma}+\left(\sum_{j=1}^{n} \mathbf{E}\left\|\xi_{j}\right\|^{2}\right)^{\gamma / 2}\right), \quad 0<\gamma<\infty,
$$

with $a_{14}$ depending on $\gamma$ only.

This Rosenthal type inequality easily follows from a result of [9] and the fact that the $\mathbf{R}^{d}$ is a type 2 space, that is, $\mathbf{E}\left\|\sum_{j=1}^{n} \xi_{j}\right\|^{2} \leqslant c \sum_{j=1}^{n} \mathbf{E}\left\|\xi_{j}\right\|^{2}$.

2. Proofs. For any random vector $\xi$ we shall denote by $\xi^{[u]}$ its truncation on the level $u>0$ :

$$
\xi^{[u]}= \begin{cases}\xi, & \text { if }\|\xi\| \leqslant u \\ 0, & \text { otherwise. }\end{cases}
$$

Set

$$
\xi^{(u)}=\xi-\xi^{[u]}, \quad \xi^{\{u\}}=\xi^{[u]}-\mathbf{E} \xi^{[u]} .
$$

Assuming $\mathbf{E}\|\xi\|^{2}<\infty$ and $\mathbf{E} \xi=0$, we have $\xi=\xi^{(u)}+\xi^{[u]}$,

$$
\mathbf{E} \xi^{(u)}+\mathbf{E} \xi^{[u]}=\mathbf{E} \xi=0,
$$

and, hence,

$$
\left\|\mathbf{E} \xi^{(u)}\right\|=\left\|\mathbf{E} \xi^{[u]}\right\| \leqslant \mathbf{E}\left\|\xi^{(u)}\right\| .
$$

Moreover, for any $v \in \mathbf{R}^{d}$,

$$
\left|\mathbf{E}\left\langle\xi^{(u)}, v\right\rangle\right|=\left|\mathbf{E}\left\langle\xi^{[u]}, v\right\rangle\right| \leqslant\|v\| \mathbf{E}\left\|\xi^{(u)}\right\| .
$$

Furthermore,

$$
\mathbf{D}\langle\xi, v\rangle=\mathbf{E}\langle\xi, v\rangle^{2}=\mathbf{E}\left\langle\xi^{[u]}, v\right\rangle^{2}+\mathbf{E}\left\langle\xi^{(u)}, v\right\rangle^{2} \geqslant \mathbf{D}\left\langle\xi^{[u]}, v\right\rangle+\mathbf{E}\left\langle\xi^{(u)}, v\right\rangle^{2} .
$$


By (2.3), (2.4) and by the Cauchy-Bunyakovskii inequality, we have

$$
\begin{aligned}
\mathbf{D}\left\langle\xi^{[u]}, v\right\rangle & =\mathbf{E}\left\langle\xi^{[u]}, v\right\rangle^{2}-\left(\mathbf{E}\left\langle\xi^{[u]}, v\right\rangle\right)^{2}=\mathbf{D}\langle\xi, v\rangle-\mathbf{E}\left\langle\xi^{(u)}, v\right\rangle^{2}-\left(\mathbf{E}\left\langle\xi^{(u)}, v\right\rangle\right)^{2} \\
& \geqslant \mathbf{D}\langle\xi, v\rangle-2 \mathbf{E}\left\langle\xi^{(u)}, v\right\rangle^{2} .
\end{aligned}
$$

Inequalities (2.4) and (2.5) imply

$$
2 \mathbf{E}\left\langle\xi^{(u)}, v\right\rangle^{2} \geqslant \mathbf{D}\langle\xi, v\rangle-\mathbf{D}\left\langle\xi^{[u]}, v\right\rangle \geqslant \mathbf{E}\left\langle\xi^{(u)}, v\right\rangle^{2} .
$$

Pr o of of $\mathrm{Th}$ e or e m 4 . The symbols $c, c_{2}, c_{1}, \ldots$ will be used for absolute positive constants. The letter $c$ can denote different constants when we do not need to fix their numerical values. The same notation will be used for positive quantities which depend only on $\alpha, \gamma, C_{1}, C_{2}$, and $C_{3}$ involved in the conditions of Theorem 4. This means, in fact, that we assume $\alpha, \gamma$, $C_{1}, C_{2}$, and $C_{3}$ to be absolute positive constants. We shall write $A \ll B$, if there exists a $c$ such that $A \leqslant c B$.

Let $\xi_{1}, \ldots, \xi_{n}$ and $\zeta_{1}, \ldots, \zeta_{s}$ be random vectors satisfying the conditions of Theorem 4. For $u>0$, consider independent Gaussian random vectors $\eta_{1}^{\{u\}}, \ldots, \eta_{n}^{\{u\}}$ with

$\mathbf{E} \eta_{k}^{\{u\}}=\mathbf{E} \zeta_{k}^{\{u\}}=0, \quad \operatorname{cov} \eta_{k}^{\{u\}}=\operatorname{cov} \zeta_{k}^{\{u\}}=\operatorname{cov} \zeta_{k}^{[u]} \stackrel{\text { def }}{=} \mathbb{B}_{k}^{\{u\}}, \quad k=1, \ldots, s$.

According to (1.11) and (2.6), for any $v \in \mathbf{R}^{d}$, we have

$$
2 \mathbf{E}\left\langle\zeta_{k}^{(u)}, v\right\rangle^{2} \geqslant\left\langle\mathbb{B}_{k} v, v\right\rangle-\left\langle\mathbb{B}_{k}^{\{u\}} v, v\right\rangle \geqslant \mathbf{E}\left\langle\zeta_{k}^{(u)}, v\right\rangle^{2}, \quad k=1, \ldots, s
$$

Thus, the operators

$$
\mathbb{B}_{k}-\mathbb{B}_{k}^{\{u\} \stackrel{\text { def }}{=}} \mathbb{B}_{k}^{(u)}
$$

are positive definite and may be treated as covariance operators. Therefore, we can construct the Gaussian vectors $\eta_{k}^{(u)}$ with $\mathbf{E} \eta_{k}^{(u)}=0$, cov $\eta_{k}^{(u)}=\mathbb{B}_{k}^{(u)}$, such that they are jointly independent and independent of the vectors $\eta_{k}^{\{u\}}$. Denote $\eta_{k}=\eta_{k}^{(u)}+\eta_{k}^{\{u\}}$. Clearly,

$$
\mathbf{E} \eta_{k}=0, \quad \operatorname{cov} \eta_{k}=\mathbb{B}_{k} .
$$

Using the standard tool of strong approximation, namely, Lemma A of [2], we can take $\xi_{j}$ as $X_{j}$ in the proof of Theorem 4, choosing below the joint distribution of $\left\{\zeta_{k}^{\{u\}}\right\}$ and $\left\{\eta_{k}^{\{u\}}\right\}$ in a special way. Moreover, we can assume that

$$
\eta_{k}=\sum_{j=m_{k-1}+1}^{m_{k}} Y_{j}, \quad k=1, \ldots, s,
$$

where $Y_{1}, \ldots, Y_{n}$ are the independent random vectors satisfying the conditions of Theorem 4.

Denoting by $\mathbb{B}^{(u)}$ the covariance operator of the sum $\sum_{k=1}^{s} \eta_{k}^{(u)}$, we have

$$
\mathbb{B}^{(u)}=\sum_{k=1}^{s} \mathbb{B}_{k}^{(u)} .
$$


Let the quantity $B^{2}(u)$ be defined by

$$
B^{2}(u) \stackrel{\text { def }}{=} \sum_{k=1}^{s} \mathbf{E}\left\|\zeta_{k}^{(u)}\right\|^{2} .
$$

By (2.7)-(2.11), for any $v \in \mathbf{R}^{d}$, the inequalities

$$
\sum_{k=1}^{s} \mathbf{E}\left\langle\zeta_{k}^{(u)}, v\right\rangle^{2} \leqslant\left\langle\mathbb{B}^{(u)} v, v\right\rangle \leqslant 2 \sum_{k=1}^{s} \mathbf{E}\left\langle\zeta_{k}^{(u)}, v\right\rangle^{2} \leqslant 2 B^{2}(u)\|v\|^{2} .
$$

are valid. Let $\sigma_{u}^{2}$ be the maximal eigenvalue of the operator $\mathbb{B}^{(u)}$. By (2.12),

$$
\sigma_{u}^{2}=\max _{\|v\|=1}\left\langle\mathbb{B}^{(u)} v, v\right\rangle \leqslant 2 B^{2}(u) .
$$

Let $Z, Z_{1}, \ldots, Z_{d}$ be i.i.d. standard normal random variables. Using standard tools to obtain exponential inequalities, it is easy to show that there exists an absolute constant $c$ such that, for any $x>c \sigma_{u} \sqrt{d}$,

$$
\mathbf{P}\left\{\left\|\sum_{k=1}^{s} \eta_{k}^{(u)}\right\| \geqslant x\right\} \leqslant \mathbf{P}\left\{\sigma_{u}^{2} \sum_{k=1}^{d} Z_{k}^{2} \geqslant x^{2}\right\} \leqslant \exp \left(-\frac{x^{2}}{8 \sigma_{u}^{2}}\right) .
$$

It suffices to note that $\mathbf{E} e^{h Z^{2}}=(1-2 h)^{-1 / 2}$, for $0<h<\frac{1}{2}$, and to apply the exponential Chebyshev inequality, choosing $h=\frac{1}{6}$.

Replacing $n$ by $s$ in the definition (1.1) of $\Delta(\cdot, \cdot)$, introduce the notation

$$
\Delta=\Delta(\zeta, \eta), \quad \Delta^{\{u\}}=\Delta\left(\zeta^{\{u\}}, \eta^{\{u\}}\right), \quad \Delta_{u}=\Delta\left(\zeta-\mathbf{E} \zeta^{[u]}, \eta^{\{u\}}\right) .
$$

It is easy to see that

$$
\Delta \leqslant \Delta_{u}+\delta_{u}+A(u)
$$

where the quantity $A(u)$ is defined by

$$
A(u) \stackrel{\text { def }}{=} \max _{1 \leqslant m \leqslant s}\left\|\sum_{k=1}^{m} \mathbf{E} \zeta_{k}^{[u]}\right\|=\max _{1 \leqslant m \leqslant s}\left\|\sum_{k=1}^{m} \mathbf{E} \zeta_{k}^{(u)}\right\|,
$$

and

$$
\delta_{u}=\Delta\left(\eta, \eta^{\{u\}}\right)
$$

Following [27], introduce the class $\mathscr{G}(u), u \geqslant 0$, of positive function $G(\cdot)$ such that the functions $G(x)$ and $x / \ln G(x)$ are nondecreasing for $x>u$. For example, the functions $G(x)=x^{\gamma}, \gamma>0$, and $\exp \left(\lambda x^{\beta}\right), 0 \leqslant \beta \leqslant 1$, $\lambda>0$, belong to $\mathscr{G}(e)$ and $\mathscr{G}(0)$, respectively.

The formulation and proof of the following lemma almost coincide with the formulation and proof of Theorem 3 in [27]. One can find it also in [36] (with $\left\{\zeta_{k}\right\}$ replaced by $\left\{\xi_{j}\right\}$ ). 
Lemma 4. Let $u>0, G(\cdot) \in \mathscr{G}(u)$, and

$$
F(x) \stackrel{\text { def }}{=} \sum_{k=1}^{s} \mathbf{P}\left\{\left\|\zeta_{k}\right\| \geqslant x\right\} \leqslant \frac{1}{G(x)}, \quad \text { for all } \quad x>u .
$$

Then, for all $x_{0} \geqslant 0$ and $x \geqslant z \geqslant u$,

$$
\mathbf{P}\left\{\Delta_{u} \geqslant x_{0}+x\right\} \leqslant \mathbf{P}\left\{\Delta^{\{u\}} \geqslant x_{0}\right\}+\mathbf{P}\left\{\max _{1 \leqslant k \leqslant s}\left\|\zeta_{k}\right\| \geqslant z\right\}+\frac{e G^{1-x / z}(z)}{G(u)} .
$$

It is easy to see that, for $k=1, \ldots, s, x \geqslant 0$,

$$
F_{k}(x) \stackrel{\text { def }}{=} \mathbf{P}\left\{\left\|\zeta_{k}\right\| \geqslant x\right\} \leqslant T_{k}(x) \stackrel{\text { def }}{=} \max _{m_{k-1}+1 \leqslant m \leqslant m_{k}} \mathbf{P}\left\{\left\|\sum_{j=m_{k-1}+1}^{m} \xi_{j}\right\| \geqslant x\right\} .
$$

It is clear that

$$
F(x)=\sum_{k=1}^{s} F_{k}(x) \leqslant \sum_{k=1}^{s} T_{k}(x)
$$

(see (2.19) and (2.21)).

We shall use the following Fuk-Nagaev type inequalities:

$$
\begin{aligned}
F_{k}(x) \leqslant & T_{k}(x) \leqslant 2 d \exp \left(-\frac{c_{1} x^{2}}{d w^{2}}\right) \\
& +2 d(2 b)^{b(\gamma-1)}\left(d^{\gamma / 2} \sum_{j=m_{k-1}+1}^{m_{k}} \frac{\mathbf{E}\left\|\xi_{j}\right\|^{\gamma}}{x^{\gamma}}\right)^{b} \\
& +d \sum_{j=m_{k-1}+1}^{m_{k}} \mathbf{P}\left(\left\|\xi_{j}\right\| \geqslant \frac{x}{2 b \sqrt{d}}\right), \quad k=1, \ldots, s, \\
F_{k}(x) \leqslant & T_{k}(x) \ll d \exp \left(-\frac{c_{1} x^{2}}{d w^{2}}\right) \\
& +d^{1+\gamma / 2} \sum_{j=m_{k-1}+1}^{m_{k}} \frac{\mathbf{E}\left\|\xi_{j}\right\|^{\gamma}}{x^{\gamma}}, \quad k=1, \ldots, s,
\end{aligned}
$$

which hold for any $x>0$ and $b \geqslant 1$. They follow from (2.21) and from the corresponding one-dimensional inequalities (1.27) and (1.28). Constants depend here on $\gamma$ and $C_{1}$, but recall that we treat $\gamma$ and $C_{1}$ as universal constants. Inequality (2.24) is weaker than (2.23), but it is more convenient in some applications below.

It should be mentioned that inequality (2.24) with constants which do not depend on the dimension $d$ was proved for $\gamma=3$ in [11], see [21, Theorem 4.3]. Therefore, for $\gamma=3$, the dependence of constants on the dimension in inequality (1.18) of Theorem 4 might be sharpened. 
We shall choose

$$
u=c_{2} d L_{\gamma}^{1 / \gamma},
$$

where the constant $c_{2}$ will be so large as it will be required below.

Using (1.14) and (2.23)-(2.25), we get that, for $x \geqslant u, b \geqslant 1$,

$$
\begin{gathered}
\exp \left(-\frac{c_{1} x^{2}}{2 d w^{2}}\right) \ll \frac{1}{2 d s}, \\
T_{k}(x) \leqslant \frac{1}{s} \exp \left(-\frac{c_{1} x^{2}}{2 d w^{2}}\right)+2 d(2 b)^{b(\gamma-1)}\left(d^{\gamma / 2} \frac{\lambda_{k, \gamma}}{x^{\gamma}}\right)^{b} \\
+d \sum_{j=m_{k-1}+1}^{m_{k}} \mathbf{P}\left\{\left\|\xi_{j}\right\| \geqslant \frac{x}{2 b \sqrt{d}}\right\}, \quad k=1, \ldots, s,
\end{gathered}
$$

and

$$
F_{k}(x) \leqslant T_{k}(x) \ll \frac{1}{s} \exp \left(-\frac{c_{1} x^{2}}{2 d w^{2}}\right)+d^{1+\gamma / 2} \frac{\lambda_{k, \gamma}}{x^{\gamma}}, \quad k=1, \ldots, s,
$$

if $c_{2}$ is large enough.

According to (1.13), (1.21), (2.19), (2.22), (2.25), and (2.28), we have

$$
F(x) \ll \exp \left(-\frac{c_{1} x^{2}}{2 d w^{2}}\right)+d^{1+\gamma / 2} \sum_{j=1}^{n} \frac{\mathbf{E}\left\|\xi_{j}\right\|^{\gamma}}{x^{\gamma}} \ll d^{1+\gamma / 2} \frac{L_{\gamma}}{x^{\gamma}},
$$

for $x \geqslant u$, that is, there exists $c_{3}$ such that

$$
F(x) \leqslant \frac{1}{G(x)}, \quad \text { where } \quad G(x)=\frac{x^{\gamma}}{c_{3} d^{1+\gamma / 2} L_{\gamma}},
$$

for $x \geqslant u$, and

$$
G(u) \geqslant e^{\gamma},
$$

if $c_{2}$ is large enough. We used here that $\gamma \geqslant 2$ and, hence, $1+\gamma / 2 \leqslant \gamma$. In order to apply Lemma 4 we have to verify that $G(\cdot) \in \mathscr{G}(u)$. Let us show that the function $x / \ln G(x)$ is nondecreasing for $x>u$. Indeed, for $x \geqslant y \geqslant u$, we have $G(y) \geqslant G(u) \geqslant e^{\gamma}$ and $G(x) / x^{\gamma}=G(y) / y^{\gamma}$. Denoting $t=x / y \geqslant 1, q=G(y)$, we get $q \geqslant e^{\gamma}, t^{\gamma} \leqslant e^{\gamma(t-1)} \leqslant q^{t-1}$ and $G(x)=q t^{\gamma} \leqslant q^{t}$. Hence, $\ln G(x) \leqslant t \ln q=(x / y) \ln G(y)$. Thus, $G(\cdot) \in \mathscr{G}(u)$.

By (2.16) and (2.20), for all $x_{0}, x_{1} \geqslant 0$ and $x \geqslant z \geqslant u$,

$$
\begin{aligned}
\mathbf{P}\left\{\Delta \geqslant x_{0}+x_{1}+x+A(u)\right\} \leqslant & \mathbf{P}\left\{\Delta_{u} \geqslant x_{0}+x\right\}+\mathbf{P}\left\{\delta_{u} \geqslant x_{1}\right\} \\
\leqslant & \mathbf{P}\left\{\Delta^{\{u\}} \geqslant x_{0}\right\}+\mathbf{P}\left\{\max _{1 \leqslant k \leqslant s}\left\|\zeta_{k}\right\| \geqslant z\right\} \\
& +\frac{e G^{1-x / z}(z)}{G(u)}+\mathbf{P}\left\{\delta_{u} \geqslant x_{1}\right\} .
\end{aligned}
$$


Using (2.18) and the Lévy inequality (see [10, Theorem 1.1.1]) and taking into account that $\eta_{k}-\eta_{k}^{\{u\}}=\eta_{k}^{(u)}$, we obtain

$$
\begin{aligned}
\mathbf{P}\left\{\delta_{u} \geqslant x_{1}\right\} & =\mathbf{P}\left\{\max _{1 \leqslant m \leqslant s}\left\|\sum_{k=1}^{m}\left(\eta_{k}-\eta_{k}^{\{u\}}\right)\right\| \geqslant x_{1}\right\} \\
& \leqslant 2 \mathbf{P}\left\{\left\|\sum_{k=1}^{s} \eta_{k}^{(u)}\right\| \geqslant x_{1}\right\} .
\end{aligned}
$$

By (2.13), (2.14), and (2.33), we have

$$
\mathbf{P}\left(\delta_{u} \geqslant x_{1}\right) \leqslant 2 \exp \left(-\frac{x_{1}^{2}}{16 B^{2}(u)}\right),
$$

for any $x_{1}>c B(u) \sqrt{d}$, where $c$ is an absolute constant.

Integrating by parts and using relations (1.14), (1.15), (1.21), (2.25), (2.26), and (2.28)-(2.31), we get rough bounds

$$
\begin{aligned}
\mathbf{E}\left\|\zeta_{k}^{(u)}\right\|^{2}= & -\int_{\{x>u\}} x^{2} d F_{k}(x)=u^{2} F_{k}(u)+2 \int_{\{x>u\}} x F_{k}(x) d x \\
\leqslant & c u^{2}\left(\frac{1}{s} \exp \left(-\frac{c_{1} u^{2}}{2 d w^{2}}\right)+d^{1+\gamma / 2} \frac{\lambda_{k, \gamma}}{u^{\gamma}}\right) \\
& +c \int_{\{x \geqslant u\}} x\left(\frac{1}{s} \exp \left(-\frac{c_{1} x^{2}}{2 d w^{2}}\right)+d^{1+\gamma / 2} \frac{\lambda_{k, \gamma}}{x^{\gamma}}\right) d x \\
\leqslant & \frac{w^{2}}{8 s}+\frac{c d^{1+\gamma / 2} \lambda_{k, \gamma}}{u^{\gamma-2}} \leqslant \frac{w^{2}}{4} \leqslant \frac{u^{2}}{4}, \quad k=1, \ldots, s
\end{aligned}
$$

and

$$
\begin{aligned}
B^{2}(u) & =-\int_{\{x>u\}} x^{2} d F(x)=u^{2} F(u)+2 \int_{\{x>u\}} x F(x) d x \\
& \leqslant \frac{u^{2}}{2}+\frac{c d^{1+\gamma / 2} L_{\gamma}}{u^{\gamma-2}} \leqslant u^{2},
\end{aligned}
$$

if $c_{2}$ is large enough.

By (1.13), (2.1), (2.2), (2.35) and by the Chebyshev inequality,

$$
\left\|\mathbf{E} \zeta_{k}^{(u)}\right\| \leqslant \frac{\mathbf{E}\left\|\zeta_{k}^{(u)}\right\|^{2}}{u} \leqslant \frac{w^{2}}{u} \leqslant w, \quad k=1, \ldots, s .
$$

By (2.7) and (2.35), for any $v \in \mathbf{R}^{d}$,

$$
\left\langle\mathbb{B}_{k} v, v\right\rangle-\left\langle\mathbb{B}_{k}^{\{u\}} v, v\right\rangle \leqslant 2 \mathbf{E}\left\langle\zeta_{k}^{(u)}, v\right\rangle^{2} \leqslant 2 \mathbf{E}\left\|\zeta_{k}^{\{u\}}\right\|^{2}\|v\|^{2} \leqslant \frac{w^{2}\|v\|^{2}}{2} .
$$

Using (1.12), (2.7), and (2.38), it is not difficult to make sure that, for any $v \in \mathbf{R}^{d}$,

$$
\frac{w^{2}\|v\|^{2}}{2} \leqslant\left\langle\mathbb{B}_{k}^{\{u\}} v, v\right\rangle \leqslant C_{1} w^{2}\|v\|^{2}, \quad k=1, \ldots, s .
$$


Arguing similarly to the proof of (2.14) and using (1.12), we obtain that there exists an absolute constant $c$ such that, for any $x>c w \sqrt{d C_{1}}$, $k=1, \ldots, s$,

$$
\mathbf{P}\left\{\left\|\sum_{j=m_{k-1}+1}^{m} Y_{j}\right\| \geqslant x\right\} \leqslant \exp \left(-\frac{x^{2}}{8 C_{1} w^{2}}\right), \quad m_{k-1}+1 \leqslant m \leqslant m_{k} .
$$

Therefore, for any $x>u, k=1, \ldots, s$,

$$
\mathbf{P}\left\{\left\|\sum_{j=m_{k-1}+1}^{m} Y_{j}\right\| \geqslant x\right\} \leqslant \frac{1}{s} \exp \left(-\frac{x^{2}}{16 C_{1} w^{2}}\right), \quad m_{k-1}+1 \leqslant m \leqslant m_{k},
$$

if $c_{2}$ in (2.25) is large enough.

Let

$$
w_{0}=c_{4} d w \varepsilon^{-1},
$$

where $0<\varepsilon<1$ and $c_{4}$ is large enough. Then, choosing $c_{4} \geqslant 1$, we can ensure that $w_{0} \geqslant d w \geqslant w$.

Below we shall estimate the expectation

$$
\mathbf{E}\left\|\zeta_{k}^{\{u\}}\right\|^{3} \exp \left(\left\|\zeta_{k}^{\{u\}}\right\| w_{0}^{-1}\right)=I_{1}+I_{2}, \quad k=1, \ldots, s,
$$

where

$$
I_{1}=\mathbf{E}\left\|\zeta_{k}^{\{u\}}\right\|^{3} \exp \left(\left\|\zeta_{k}^{\{u\}}\right\| w_{0}^{-1}\right) 1\left\{\left\|\zeta_{k}^{[u]}\right\|<w\right\}
$$

and

$$
I_{2}=\mathbf{E}\left\|\zeta_{k}^{\{u\}}\right\|^{3} \exp \left(\left\|\zeta_{k}^{\{u\}}\right\| w_{0}^{-1}\right) \mathbf{1}\left\{\left\|\zeta_{k}^{[u]}\right\| \geqslant w\right\} .
$$

Notation $\mathbf{1}\{A\}$ is used here for the indicator function of the event $A$. Taking into account relations $(2.2),(2.37),(2.44)$, and $w_{0} \geqslant w$, we obtain $\zeta_{k}^{\{u\}}=$ $\zeta_{k}^{[u]}-\mathbf{E} \zeta_{k}^{[u]}=\zeta_{k}^{[u]}+\mathbf{E} \zeta_{k}^{(u)}$ and

$$
I_{1} \ll w^{3} .
$$

Moreover, by (2.1), (2.37), and (2.45),

$$
\begin{aligned}
I_{2} & \leqslant \mathbf{E}\left\|\zeta_{k}+\mathbf{E} \zeta_{k}^{(u)}\right\|^{3} \exp \left(\left\|\zeta_{k}+\mathbf{E} \zeta_{k}^{(u)}\right\| w_{0}^{-1}\right) \mathbf{1}\left\{w \leqslant\left\|\zeta_{k}\right\| \leqslant u\right\} \\
& \ll \mathbf{E}\left(\left\|\zeta_{k}\right\|^{3}+w^{3}\right) \exp \left(\left\|\zeta_{k}\right\| w_{0}^{-1}\right) \mathbf{1}\left\{w \leqslant\left\|\zeta_{k}\right\| \leqslant u\right\} .
\end{aligned}
$$

Integrating by parts and using relations $(2.21)$ and $w_{0} \geqslant w$, we obtain

$$
\begin{aligned}
& \mathbf{E}\left(\left\|\zeta_{k}\right\|^{3}+w^{3}\right) \exp \left(\left\|\zeta_{k}\right\| w_{0}^{-1}\right) \mathbf{1}\left\{w \leqslant\left\|\zeta_{k}\right\| \leqslant u\right\} \\
& \quad=-\int_{\{w \leqslant x \leqslant u\}}\left(x^{3}+w^{3}\right) e^{x / w_{0}} d F_{k}(x) \\
& \quad \leqslant 2 e w^{3} F_{k}(w)+\int_{\{w \leqslant x \leqslant u\}}\left(3 x^{2}+\frac{x^{3}+w^{3}}{w_{0}}\right) e^{x / w_{0}} F_{k}(x) d x .
\end{aligned}
$$


Furthermore, by (1.14) and (2.24), we have

$$
\int_{\{w \leqslant x \leqslant u\}}\left(3 x^{2}+\frac{x^{3}+w^{3}}{w_{0}}\right) e^{x / w_{0}} F_{k}(x) d x \ll I_{3}+I_{4},
$$

where

$$
I_{3}=d \int_{\{w \leqslant x \leqslant u\}}\left(3 x^{2}+\frac{x^{3}+w^{3}}{w_{0}}\right) e^{x / w_{0}} \exp \left(-\frac{c_{1} x^{2}}{2 d w^{2}}\right) d x
$$

and

$$
I_{4}=\int_{\{w \leqslant x \leqslant u\}}\left(3 x^{2}+\frac{x^{3}+w^{3}}{w_{0}}\right) e^{x / w_{0}} d^{1+\gamma / 2} \frac{\lambda_{k, \gamma}}{x^{\gamma}} d x .
$$

It is easy to see that

$$
I_{3} \ll \int_{\{w \leqslant x\}}\left(3 x^{2}+\frac{x^{3}+w^{3}}{w_{0}}\right) \exp \left(-\frac{c_{1} x^{2}}{4 d w^{2}}\right) d x \ll d^{2} w^{3} \ll d w_{0} w^{2},
$$

if $c_{4}$ in (2.42) is sufficiently large. Furthermore, by (1.13), (1.15), (2.25), $(2.42)$, and (2.51),

$$
\begin{aligned}
I_{4} & \ll d^{1+\gamma / 2}\left(3 u^{2}+\frac{u^{3}+w^{3}}{w_{0}}\right) e^{u / w_{0}} \lambda_{k, \gamma} \int_{\{w \leqslant x\}} \frac{d x}{x^{\gamma}} \\
& \ll \frac{d^{2+\gamma / 2} \lambda_{k, \gamma} s^{\varepsilon}\left(\ln ^{*} s\right)^{\gamma+3}}{L_{\gamma}} w_{0} w^{2} \ll d^{2} w_{0} w^{2},
\end{aligned}
$$

if $c_{4}$ is large enough. Collecting bounds (2.43) and (2.46)-(2.53), we obtain

$$
\mathbf{E}\left\|\zeta_{k}^{\{u\}}\right\|^{3} \exp \left(\left\|\zeta_{k}^{\{u\}}\right\| w_{0}^{-1}\right) \ll d^{2} w_{0} w^{2} .
$$

Using (2.54), we get, for $m=3,4, \ldots$,

$$
\mathbf{E}\left\|\zeta_{k}^{\{u\}}\right\|^{m} \leqslant(m-3) ! w_{0}^{m-3} \mathbf{E}\left\|\zeta_{k}^{\{u\}}\right\|^{3} \exp \left(\left\|\zeta_{k}^{\{u\}}\right\| w_{0}^{-1}\right) \ll d^{2} m ! w_{0}^{m-2} w^{2} .
$$

By (2.39), inequality (2.55) is valid for $m=2,3, \ldots$.

Let us prove that $\mathscr{L}\left(\zeta_{k}^{\{u\}}\right) \in \mathscr{A}_{d}\left(c_{5} d^{2} w_{0}\right)$, where the constant $c_{5}$ will be chosen to be so large as it will be necessary for the arguments below. Note that, in [36], the trivial relation $\mathscr{L}\left(\zeta_{k}^{\{u\}}\right) \in \mathscr{A}_{d}(c u)$ with a universal constant $c$ was used. To simplify the formulas, denote $\xi=\zeta_{k}^{\{u\}}$. Let $z \in \mathbb{C}^{d}$, $\|z\| c_{5} w_{0} \leqslant 1, x=\operatorname{Re} z, y=\operatorname{Im} z$. Recall that $d w \leqslant w_{0}$. Choosing $c_{5}$ to be sufficiently large, and using (2.39), (2.42), and (2.55), we ensure the validity of the relations

$$
\begin{aligned}
\left\langle\mathbb{B}_{k}^{\{u\}} x, x\right\rangle+\left\langle\mathbb{B}_{k}^{\{u\}} y, y\right\rangle & \leqslant C_{1} w^{2}\left(\|x\|^{2}+\|y\|^{2}\right)=C_{1} w^{2}\|z\|^{2} \leqslant 1 \\
\left|\mathbf{E} e^{\langle z, \xi\rangle}-1\right| & =\left|\sum_{m=2}^{\infty} \frac{1}{m !} \mathbf{E}\langle z, \xi\rangle^{m}\right| \\
& \leqslant \sum_{m=2}^{\infty} \frac{2^{m}}{m !}\left(\mathbf{E}|\langle x, \xi\rangle|^{m}+\mathbf{E}|\langle y, \xi\rangle|^{m}\right)
\end{aligned}
$$




$$
\begin{aligned}
& \leqslant c \sum_{m=2}^{\infty} 2^{m}\left(\left(\|x\| w_{0}\right)^{m-2}+\left(\|y\| w_{0}\right)^{m-2}\right) d^{2} w^{2}\|z\|^{2} \\
& \leqslant 16 c w_{0}^{2}\|z\|^{2} \leqslant \frac{1}{2}
\end{aligned}
$$

and, consequently,

$$
\frac{1}{2} \leqslant\left|\mathbf{E} e^{\langle z, \xi\rangle}\right| \leqslant \frac{3}{2}
$$

Choosing $c_{5}$ to be sufficiently large, expanding the exponent and using (2.55), we see that

$$
\mathbf{E}\langle\xi, v\rangle^{2} e^{2|\langle\xi, x\rangle|} \ll d^{2} w^{2}\|v\|^{2}, \quad \text { for all } \quad v \in \mathbf{R}^{d} .
$$

Differentiating the function $\varphi(V, z)$ with $V=\mathscr{L}\left(\zeta_{k}^{\{u\}}\right)$ (see (1.8)), we obtain

$$
\begin{aligned}
d_{u} d_{v}^{2} \varphi(V, z)= & \frac{\mathbf{E}\langle\xi, u\rangle\langle\xi, v\rangle^{2} e^{\langle z, \xi\rangle} \mathbf{E} e^{\langle z, \xi\rangle}-\mathbf{E}\langle\xi, v\rangle^{2} e^{\langle z, \xi\rangle} \mathbf{E}\langle\xi, u\rangle e^{\langle z, \xi\rangle}}{\left(\mathbf{E} e^{\langle z, \xi\rangle}\right)^{2}} \\
& -\frac{2 \mathbf{E}\langle\xi, v\rangle e^{\langle z, \xi\rangle}}{\mathbf{E} e^{\langle z, \xi\rangle}} \frac{1}{\left(\mathbf{E} e^{\langle z, \xi\rangle}\right)^{2}} \\
& \times\left(\mathbf{E}\langle\xi, u\rangle\langle\xi, v\rangle e^{\langle z, \xi\rangle} \mathbf{E} e^{\langle z, \xi\rangle}-\mathbf{E}\langle\xi, v\rangle e^{\langle z, \xi\rangle} \mathbf{E}\langle\xi, u\rangle e^{\langle z, \xi\rangle}\right) .
\end{aligned}
$$

Using (2.39), (2.42), (2.55), (2.58)-(2.60), and the Hölder inequality, we have

$$
\begin{aligned}
&\left|d_{u} d_{v}^{2} \varphi(V, z)\right| \leqslant c(\left(\mathbf{E}\langle\xi, u\rangle^{2}\langle\xi, v\rangle^{2} \mathbf{E}\langle\xi, v\rangle^{2} e^{2\langle\xi, x\rangle}\right)^{1 / 2} \\
&\left.+\left(\mathbf{E}\langle\xi, v\rangle^{2}+\mathbf{E}\langle\xi, v\rangle^{2} e^{2\langle\xi, x\rangle}\right)\left(\mathbf{E}\langle\xi, u\rangle^{2} e^{2\langle\xi, x\rangle}\right)^{1 / 2}\right) \\
& \leqslant c\|u\| w_{0} d^{2} w^{2}\|v\|^{2} \leqslant c_{5} d^{2} w_{0}\|u\|\left\langle\mathbb{B}_{k}^{\{u\}} v, v\right\rangle,
\end{aligned}
$$

if $c_{5}$ is chosen to be large enough. This implies that

$$
\mathscr{L}\left(\zeta_{k}^{\{u\}}\right) \in \mathscr{A}_{d}\left(c_{5} d^{2} w_{0}\right)
$$

(see (1.9)). Using (2.42) and the properties of the classes $\mathscr{A}_{d}(\tau)$, we see that

$$
\mathscr{L}\left(\zeta_{k}^{\{u\}} w^{-1}\right) \in \mathscr{A}_{d}\left(c_{5} d^{2} w_{0} w^{-1}\right) \subset \mathscr{A}_{d}\left(c_{6} d^{3}\right)
$$

with some $c_{6} \geqslant 1$.

By Theorem 3 applied (with $\tau=c_{6} d^{3}$ ) to the vectors $\zeta_{k}^{\{u\}} / w$, there exists a construction such that the joint distribution of $\left\{\zeta_{k}^{\{u\}}\right\}$ and $\left\{\eta_{k}^{\{u\}}\right\}$ satisfies

$$
\mathbf{E} \exp \left(\frac{c_{7} \Delta^{\{u\}}}{w d^{15 / 2} \ln ^{*} d}\right)=\mathbf{E} \exp \left(\frac{c_{8} \varepsilon \Delta^{\{u\}} \ln ^{*} s}{L_{\gamma}^{1 / \gamma} d^{15 / 2} \ln ^{*} d}\right) \leqslant \exp \left(c_{9} d^{3+\alpha} \ln ^{*} s\right),
$$

where $c_{7}, c_{8}, c_{9}$ are positive quantities depending on $\alpha, \gamma, C_{1}, C_{2}$, and $C_{3}$ only. Recall that we consider them as universal constants. Condition (1.10) 
of Theorem 3 is satisfied due to (2.39). By (2.62) and by the Chebyshev inequality,

$$
\begin{aligned}
\mathbf{P}\left\{\Delta^{\{u\}} \geqslant x_{0}\right\} & \leqslant \exp \left(c_{9} d^{3+\alpha} \ln ^{*} s-\frac{c_{8} \varepsilon x_{0} \ln ^{*} s}{L_{\gamma}^{1 / \gamma} d^{15 / 2} \ln ^{*} d}\right) \\
& \leqslant \exp \left(-\frac{c_{8} \varepsilon x_{0} \ln ^{*} s}{2 L_{\gamma}^{1 / \gamma} d^{15 / 2} \ln ^{*} d}\right),
\end{aligned}
$$

provided that $c_{9} d^{3+\alpha}<c_{8} \varepsilon x_{0} /\left(2 L_{\gamma}^{1 / \gamma} d^{15 / 2} \ln ^{*} d\right)$.

Using (2.32), (2.34), and (2.63), we get

$$
\begin{aligned}
\mathbf{P}\{\Delta & \left.\geqslant x_{0}+x_{1}+x+A(u)\right\} \\
\leqslant & 2 \exp \left(-\frac{x_{1}^{2}}{16 B^{2}(u)}\right)+\exp \left(-\frac{c_{8} \varepsilon x_{0} \ln ^{*} s}{2 L_{\gamma}^{1 / \gamma} d^{15 / 2} \ln ^{*} d}\right) \\
& +\mathbf{P}\left\{\max _{1 \leqslant k \leqslant s}\left\|\zeta_{k}\right\| \geqslant z\right\}+\frac{e G^{1-x / z}(z)}{G(u)},
\end{aligned}
$$

for $x \geqslant z \geqslant u$ and $x_{0}>2 L_{\gamma}^{1 / \gamma} c_{9} c_{8}^{-1} \varepsilon^{-1} d^{21 / 2+\alpha} \ln ^{*} d, x_{1}>c B(u) \sqrt{d}$.

We shall apply this inequality in the case, where $G(x)$ is defined in $(2.30)$ and

$$
u \leqslant z=x_{0}=x_{1} \leqslant x=2 z .
$$

By (2.1), (2.2), (2.11), (2.17), (2.30), (2.36), and (2.65), we have

$$
\begin{gathered}
A(u) u^{-1} \leqslant B^{2}(u) u^{-2} \leqslant 1, \\
\mathbf{P}\left\{\max _{1 \leqslant k \leqslant s}\left\|\zeta_{k}\right\| \geqslant z\right\} \leqslant \sum_{k=1}^{s} \mathbf{P}\left\{\left\|\zeta_{k}\right\| \geqslant z\right\}=F(z) \ll d^{1+\gamma / 2} \frac{L_{\gamma}}{z^{\gamma}} .
\end{gathered}
$$

Recall that $G(u) \geqslant e^{\gamma}$ (see (2.31)). Finally, by (2.64)-(2.67),

$$
\begin{aligned}
\mathbf{P}\{\Delta \geqslant 5 z\} & \leqslant \mathbf{P}\{\Delta \geqslant 4 z+A(u)\}=\mathbf{P}\left\{\Delta \geqslant x_{0}+x_{1}+x+A(u)\right\} \\
& \leqslant 2 \exp \left(-\frac{z^{2}}{16 u^{2}}\right)+\exp \left(-\frac{c_{10} \varepsilon z \ln ^{*} s}{L_{\gamma}^{1 / \gamma} d^{15 / 2} \ln ^{*} d}\right)+2 F(z),
\end{aligned}
$$

provided that

$$
z>z_{0} \stackrel{\text { def }}{=} c_{11} \varepsilon^{-1} L_{\gamma}^{1 / \gamma} d^{21 / 2+\alpha} \ln ^{*} d \geqslant d^{9} u \geqslant u,
$$

where $c_{10}, c_{11}$ are positive quantities depending on $\alpha, \gamma, C_{1}, C_{2}$, and $C_{3}$ only. The $c_{11}$ will be chosen to be sufficiently large. Define

$$
\begin{aligned}
\Delta^{*} & =\max _{1 \leqslant k \leqslant s} \max _{m_{k-1}+1 \leqslant m \leqslant m_{k}}\left\|\sum_{j=m_{k-1}+1}^{m} \xi_{j}\right\|, \\
\Delta^{* *} & =\max _{1 \leqslant k \leqslant s} \max _{m_{k-1}+1 \leqslant m \leqslant m_{k}}\left\|\sum_{j=m_{k-1}+1}^{m} Y_{j}\right\| .
\end{aligned}
$$


Then, according to (1.11), (2.9), (2.15), (2.70), and (2.71), we have, for any $z \geqslant 0$,

$$
\mathbf{P}\{\Delta(\xi, Y) \geqslant 9 z\} \leqslant \mathbf{P}\{\Delta \geqslant 5 z\}+\mathbf{P}\left\{\Delta^{*} \geqslant 3 z\right\}+\mathbf{P}\left\{\Delta^{* *} \geqslant z\right\} .
$$

To bound the second summand in (2.72), we apply Lemma 1. By this lemma, we derive

$$
\begin{aligned}
\mathbf{P}\left\{\Delta^{*} \geqslant 3 z\right\} & \leqslant \sum_{k=1}^{s} \mathbf{P}\left\{\max _{m_{k-1}+1 \leqslant m \leqslant m_{k}}\left\|\sum_{j=m_{k-1}+1}^{m} \xi_{j}\right\| \geqslant 3 z\right\} \\
& \leqslant 3 \sum_{k=1}^{s} \max _{m_{k-1}+1 \leqslant m \leqslant m_{k}} \mathbf{P}\left\{\left\|\sum_{j=m_{k-1}+1}^{m} \xi_{j}\right\| \geqslant z\right\} \\
& =3 \sum_{k=1}^{s} T_{k}(z)
\end{aligned}
$$

(see (2.21)). By (2.41) and by the Lévy inequality,

$$
\begin{aligned}
\mathbf{P}\left\{\Delta^{* *} \geqslant z\right\} & \leqslant \sum_{k=1}^{s} \mathbf{P}\left\{\max _{m_{k-1}+1 \leqslant m \leqslant m_{k}}\left\|\sum_{j=m_{k-1}+1}^{m} Y_{j}\right\| \geqslant z\right\} \\
& \leqslant 2 \sum_{k=1}^{s} \mathbf{P}\left\{\left\|\sum_{j=m_{k-1}+1}^{m_{k}} Y_{j}\right\| \geqslant z\right\} \\
& \leqslant 2 \exp \left(-\frac{z^{2}}{16 C_{1} w^{2}}\right),
\end{aligned}
$$

for $z>u$. Collecting bounds (2.22), (2.67), (2.68), and (2.72)-(2.74), we obtain

$$
\begin{aligned}
\mathbf{P}\{\Delta(\xi, Y) \geqslant 9 z\} \leqslant & 5 \sum_{k=1}^{s} T_{k}(z)+2 \exp \left(-\frac{z^{2}}{16 C_{1} w^{2}}\right) \\
& +2 \exp \left(-\frac{z^{2}}{16 u^{2}}\right)+\exp \left(-\frac{c_{10} \varepsilon z \ln ^{*} s}{L_{\gamma}^{1 / \gamma} d^{15 / 2} \ln ^{*} d}\right)
\end{aligned}
$$

provided that $z>z_{0}$. Relations (1.13), (1.21), (2.25), (2.27), and (2.75) imply inequality (1.16).

Choosing $c_{11}$ to be large enough and using (2.69) and the inequality $r^{\beta} \beta^{-\beta} \leqslant e^{r}$, we obtain the rough bound

$$
\begin{aligned}
\exp \left(-\frac{c_{10} \varepsilon z}{L_{\gamma}^{1 / \gamma} d^{15 / 2} \ln ^{*} d}\right) \leqslant & \left(\frac{2 L_{\gamma}^{1 / \gamma} d^{15 / 2} \ln ^{*} d \cdot \gamma b}{c_{10} \varepsilon z}\right)^{\gamma b} \\
& \times \exp \left(-\frac{c_{10} \varepsilon z}{2 L_{\gamma}^{1 / \gamma} d^{15 / 2} \ln ^{*} d}\right) \\
\leqslant & \left(2 c_{10}^{-1} \gamma b \varepsilon^{-1} d^{15 / 2} \ln ^{*} d\right)^{\gamma b}\left(L_{\gamma} z^{-\gamma}\right)^{b} e^{-d^{3}}
\end{aligned}
$$


for any $b \geqslant 1$ and $z>z_{0}$. Moreover, $\left(d^{9}\right)^{\gamma b} \leqslant(3 \gamma b)^{3 \gamma b} e^{d^{3}}$, and, hence, there exists $c_{13}$ such that

$$
\exp \left(-\frac{c_{10} \varepsilon z}{L_{\gamma}^{1 / \gamma} d^{15 / 2} \ln ^{*} d}\right) \leqslant\left(c_{13} \gamma^{4} b^{4} \varepsilon^{-1}\right)^{\gamma b}\left(L_{\gamma} z^{-\gamma}\right)^{b},
$$

for any $b \geqslant 1$ and $z>z_{0}$. In a similar way one can estimate the other exponential terms in (2.75). Using (1.14), (1.15), and (1.21), we get

$$
\sum_{k=1}^{s} \lambda_{k, \gamma}^{b} \leqslant L_{\gamma}\left(\max _{1 \leqslant k \leqslant s} \lambda_{k, \gamma}\right)^{b-1} \leqslant \frac{L_{\gamma}^{b}}{\left(C_{3} d^{\gamma / 2} s^{\varepsilon}\left(\ln ^{*} s\right)^{\gamma+3}\right)^{b-1}},
$$

for $b \geqslant 1$. Relations (1.13), (2.25), (2.27), and (2.75)-(2.77) imply inequality (1.17). Inequality (1.18) may be easily deduced from (1.17) with $b=1$ estimating the last sum by the Chebyshev inequality.

Integrating by parts, we get

$$
\mathbf{E}\left(\frac{\Delta(\xi, Y)}{9}\right)^{\gamma}=\gamma \int_{0}^{\infty} z^{\gamma-1} \mathbf{P}\{\Delta(\xi, Y) \geqslant 9 z\} d z=\gamma\left(K_{1}+K_{2}\right),
$$

where

$$
K_{1}=\int_{0}^{z_{0}} z^{\gamma-1} \mathbf{P}\{\Delta(\xi, Y) \geqslant 9 z\} d z
$$

and

$$
K_{2}=\int_{z_{0}}^{\infty} z^{\gamma-1} \mathbf{P}\{\Delta(\xi, Y) \geqslant 9 z\} d z
$$

It is easy to see that, by (2.69) and (2.79),

$$
K_{1} \leqslant \int_{0}^{z_{0}} z^{\gamma-1} d z=\frac{z_{0}^{\gamma}}{\gamma} \ll L_{\gamma}\left(\varepsilon^{-1} d^{21 / 2+\alpha} \ln ^{*} d\right)^{\gamma} .
$$

Applying inequality (1.17) with $b=2$, we see that

$$
K_{2} \ll J_{1}+J_{2}
$$

where

$$
\begin{aligned}
& J_{1}=d^{1+\gamma / 2} \varepsilon^{-2 \gamma} L_{\gamma}^{2} \int_{z_{0}}^{\infty} \frac{d z}{z^{\gamma+1}} \\
& J_{2}=d \int_{z_{0}}^{\infty} z^{\gamma-1} \sum_{j=1}^{n} \mathbf{P}\left\{\left\|\xi_{j}\right\| \geqslant \frac{z}{4 \sqrt{d}}\right\} d z .
\end{aligned}
$$

Using (2.69) and (2.83), we see that

$$
J_{1} \ll d^{1+\gamma / 2} \varepsilon^{-2 \gamma} L_{\gamma}^{2} z_{0}^{-\gamma} \ll \varepsilon^{-\gamma} L_{\gamma} .
$$

Moreover,

$$
\int_{z_{0}}^{\infty} z^{\gamma-1} \mathbf{P}\left\{\left\|\xi_{j}\right\| \geqslant \frac{z}{4 \sqrt{d}}\right\} d z \ll \mathbf{E}\left(4 \sqrt{d}\left\|\xi_{j}\right\|\right)^{\gamma} \ll d^{\gamma / 2} \mathbf{E}\left\|\xi_{j}\right\|^{\gamma}
$$


Using (1.5), (2.84), and (2.86), we obtain

$$
J_{2} \ll d^{1+\gamma / 2} L_{\gamma} .
$$

Collecting relations (2.78)-(2.82), (2.85), and (2.87), we obtain

$$
\mathbf{E}(\Delta(\xi, Y))^{\gamma} \ll L_{\gamma}\left(\varepsilon^{-1} d^{21 / 2+\alpha} \ln ^{*} d\right)^{\gamma},
$$

proving (1.19). The proof of Theorem 4 is thus completed.

Proof of Proposition 1. Similarly to the proof of Theorem 4, we shall treat $\gamma$ and $C_{4}$ as absolute positive constants.

Define

$$
\begin{aligned}
& \Delta_{1}=\max _{1 \leqslant m \leqslant n}\left\|\sum_{j=1}^{m} X_{j}\right\|, \\
& \Delta_{2}=\max _{1 \leqslant m \leqslant n}\left\|\sum_{j=1}^{m} Y_{j}\right\|,
\end{aligned}
$$

Then, according to (1.1), (2.89), and (2.90), we have, for any $z \geqslant 0$,

$$
\mathbf{P}\{\Delta(X, Y) \geqslant 4 z\} \leqslant \mathbf{P}\left\{\Delta_{1} \geqslant 3 z\right\}+\mathbf{P}\left\{\Delta_{2} \geqslant z\right\} .
$$

To bound the first summand, we apply Lemma 1. By this lemma, we derive

$$
\mathbf{P}\left\{\Delta_{1} \geqslant 3 z\right\} \leqslant 3 \max _{1 \leqslant m \leqslant n} \mathbf{P}\left\{\left\|\sum_{j=1}^{m} X_{j}\right\| \geqslant z\right\}
$$

(see (2.89)). By (2.90) and by the Lévy inequality,

$$
\mathbf{P}\left\{\Delta_{2} \geqslant z\right\} \leqslant 2 \mathbf{P}\left\{\left\|\sum_{j=1}^{n} Y_{j}\right\| \geqslant z\right\} .
$$

Applying Lemma 2 and using (1.5), we get, similarly to (2.23), that, for any $z>0$ and $b \geqslant 1$,

$$
\begin{aligned}
\mathbf{P}\left\{\left\|\sum_{j=1}^{m} X_{j}\right\| \geqslant z\right\} \leqslant & 2 d \exp \left(-\frac{c_{12} z^{2}}{d \sigma^{2}}\right)+2(2 b)^{b(\gamma-1)} d^{1+\gamma b / 2} \frac{L_{\gamma}^{b}}{z^{b \gamma}} \\
& +d \sum_{j=1}^{n} \mathbf{P}\left\{\left\|X_{j}\right\| \geqslant \frac{z}{2 b \sqrt{d}}\right\}, \quad m=1, \ldots, n .
\end{aligned}
$$

Integrating by parts and using (2.91), we get

$$
\mathbf{E}\left(\frac{\Delta(X, Y)}{4}\right)^{\gamma}=\gamma \int_{0}^{\infty} z^{\gamma-1} \mathbf{P}\{\Delta(X, Y) \geqslant 4 z\} d z=\gamma\left(M_{1}+M_{2}+M_{3}\right),
$$


where

$$
\begin{aligned}
& M_{1}=\int_{0}^{y} z^{\gamma-1} \mathbf{P}\{\Delta(X, Y) \geqslant 4 z\} d z \\
& M_{2}=\int_{y}^{\infty} z^{\gamma-1} \mathbf{P}\left\{\Delta_{1} \geqslant 3 z\right\} d z \\
& M_{3}=\int_{y}^{\infty} z^{\gamma-1} \mathbf{P}\left\{\Delta_{2} \geqslant z\right\} d z
\end{aligned}
$$

with $y=c_{13} \sqrt{d} L_{\gamma}^{1 / \gamma}$ and sufficiently large constant $c_{13}$. It is easy to see that

$$
M_{1} \leqslant \int_{0}^{y} z^{\gamma-1} d z=\frac{y^{\gamma}}{\gamma} \ll d^{\gamma / 2} L_{\gamma}
$$

To estimate $M_{2}$, we shall apply inequality (2.94) with $b=2$. It is clear that

$$
\int_{y}^{\infty} z^{\gamma-1} \exp \left(-\frac{c_{12} z^{2}}{d \sigma^{2}}\right) d z \ll d^{\gamma / 2} \sigma^{\gamma} \leqslant d^{\gamma / 2} L_{\gamma}
$$

(see (1.22)) and

$$
\int_{y}^{\infty} z^{\gamma-1} \frac{L_{\gamma}^{2}}{z^{2 \gamma}} d z \ll L_{\gamma}^{2} y^{-\gamma} \ll d^{-\gamma / 2} L_{\gamma}
$$

Moreover,

$$
\int_{y}^{\infty} z^{\gamma-1} \mathbf{P}\left\{\left\|\xi_{j}\right\| \geqslant \frac{z}{4 \sqrt{d}}\right\} d z \ll \mathbf{E}\left(4 \sqrt{d}\left\|\xi_{j}\right\|\right)^{\gamma}=4^{\gamma} d^{\gamma / 2} \mathbf{E}\left\|\xi_{j}\right\|^{\gamma}
$$

and, by (1.5), (2.92), (2.94), (2.97), and (2.100)-(2.102),

$$
M_{2} \ll d^{1+\gamma / 2} L_{\gamma}
$$

Arguing similarly to the proof of (2.14) and using the definition of $\sigma^{2}$, we obtain that there exists an absolute constant $c_{14}$ such that, for any $z>c_{14} \sigma \sqrt{d}$,

$$
\mathbf{P}\left\{\left\|\sum_{j=1}^{n} Y_{j}\right\| \geqslant z\right\} \leqslant \exp \left(-\frac{z^{2}}{8 \sigma^{2}}\right) \text {. }
$$

Choosing $c_{13} \geqslant c_{14} C_{4}$ and using (1.22), we have $y \geqslant c_{14} \sigma \sqrt{d}$. By (1.22), (2.93), (2.98), and (2.104), we get

$$
M_{3} \ll \sigma^{\gamma} \leqslant L_{\gamma}
$$

Collecting relations $(2.95),(2.99),(2.103)$, and (2.105), we obtain

$$
\mathbf{E}\left(\frac{\Delta(X, Y)}{4}\right)^{\gamma} \ll d^{1+\gamma / 2} L_{\gamma}
$$

proving (1.24). Note that a similar inequality for $\left\|\sum_{j=1}^{n} X_{j}-\sum_{j=1}^{n} Y_{j}\right\|$ instead of $\Delta(X, Y)$ might easily be derived from Lemma 3. Inequality (1.25) follows from (1.24) by the Chebyshev inequality. 
By (1.22), there exists $c_{15}$ such that

$$
\exp \left(-\frac{c_{12} z^{2}}{d \sigma^{2}}\right)+\exp \left(-\frac{z^{2}}{8 \sigma^{2}}\right) \leqslant\left(c_{15} \gamma b d\right)^{b \gamma / 2} \frac{L_{\gamma}^{b}}{z^{b \gamma}}
$$

Inequality (1.23) follows from (2.91)-(2.94), (2.104), and (2.107). The proof of Proposition 1 is thus completed.

\section{СПИСОК ЛИТЕРАТУРЫ}

1. Berger E. Fast sichere Approximation von Partialsummen unabhängiger und stationärer ergodischer Folgen von Zufallsvectoren. Dissertation. Universität Göttingen, 1982.

2. Berkes I., Philipp W. Approximation theorems for independent and weakly dependent random vectors. - Ann. Probab., 1979, v. 7, № 1, p. 29-54.

3. Боровков A.A. О скорости сходимости в принципе инвариантности. - Теория вероятн. и ее примен., 1973., т. 18 , в. 2 , с. 217-234.

4. Боровков A.A., Саханенко А.И. Об оценках скорости сходимости в принципе инвариатности для банаховых пространств. - Теория вероятн. и ее примен., 1980 , т. XXV, в. 4, с. $734-744$.

5. Borovkov A.A., Sakhanenko A.I. On the rate of convergence in invariance principle. - Lecture Notes in Math., 1983, v. 1021, p. 59-66.

6. Csörgö M., Révész P. A new method to prove Strassen type laws of invariance principle. I, II. - Z. Wahrscheinlichkeitstheor. verw. Geb., 1974/75, v. 31, p. 255-259; p. $261-269$.

7. Csörgő M., Révész P. Strong Approximations in Probability and Statistics. New York: Academic Press, 1981, $284 \mathrm{p}$

8. Csörgö S., Hall P. The Komlós-Major-Tusnády approximations and their applications. - Austral. J. Statist., 1984, v. 26, № 2, p. 189-218.

9. de Acosta $A$. Inequalities for $B$-valued random vectors with applications to the strong law of large numbers. - Ann. Probab., 1981, v. 9, № 1, p. 157-161.

10. de la Peña V. H., Giné E. Decoupling. From dependence to independence. Randomly stopped processes. $U$-statistics and processes. Martingales and beyond. New York: Springer-Verlag, 1999, $392 \mathrm{p}$.

11. Эбралидзе $\amalg$. С. Неравенства для вероятностей больших уклонений в многомерном случае. - Теория вероятн. и ее примен., 1971, т. 16, в. 4, с. 755-759.

12. Einmahl $U$. A useful estimate in the multidimensional invariance principle. - Probab. Theory Related Fields, 1987, v. 76, № 1, p. 81-101.

13. Einmahl $U$. Strong invariance principles for partial sums of independent random vectors. - Ann. Probab., 1987, v. 15, p. 1419-1440.

14. Einmahl $U$. Extensions of results of Komlós, Major and Tusnády to the multivariate case. - J. Multivariate Anal., 1989, v. 28, p. 20-68.

15. Etemadi N. On some classical results in probability theory. - Sankhyā, Ser. A, 1985, v. 47 , № 2 , p. $215-221$.

16. Фук Д. Х., Нагаев С. В. Вероятностные неравенства для сумм независимых случайных величин. - Теория вероятн. и ее примен., 1971, т. 16, в. 4, с. 660-675 исправл.: ibid., 1976 , т. 21, в. 4.

17. Городеикий В. В. О скорости сходимости в многомерном принципе инвариантности. - Теория вероятн. и ее примен., 1975 , т. 20 , в. 3, с. $642-649$.

18. Kwapień S., Woyczynski W. A. Random Series and Stochastic Integrals: Single and Multiple. Boston: Birkhäuser, 1992, 360 p. 
19. Komlós J., Major P., Tusnády G. An approximation of partial sums of independent RV's and the sample DF. I, II. - Z. Wahrscheinlichkeitstheor. verw. Geb., 1975, v. 32, p. 111-131; 1976, v. 34, p. 33-58.

20. Major $P$. On the invariance principle for sums of independent identically distributed random variables. - J. Multivariate Anal., 1978, v. 8, p. 487-517.

21. Nagaev S. V. Large deviations of sums of independent random variables. - Ann. Probab., 1979, v. 7, № 5, p. 745-789.

22. Philipp $W$. Almost sure invariance principles for sums of $B$-valued random variables. - Lecture Notes in Math., 1979, v. 709, p. 171-193.

23. Прохоров Ю. В. Сходимость случайных процессов и предельные теоремы теории вероятностей. - Теория вероятн. и ее примен., 1956, т. 1, в. 2, с. 177-238.

24. Rosenthal H. P. On the subspaces of $L^{p}(p>2)$ spanned by sequences of independent random variables. - Israel J. Math., 1970, v. 8, p. 273-303.

25. Rosenthal $H$. $P$. On the span in $L^{p}$ of sequences of independent random variables. II. Proceedings of the Sixth Berkeley Symposium on Mathematical Statistics and Probability (Univ. California, Berkeley, 1970/1971). V. II: Probability theory. Berkeley: Univ. California Press, 1972, p. 149-167.

26. Саханенко А.И. Скорость сходимости в принципе инвариантности для разнораспределенных величин с экпоненциальными моментами. - Тр. Ин-та матем. CO AH CCCP, 1984, т. 3, с. 4-49.

27. Саханенко А. И. Оценки в принципах инвариантности. - Тр. Ин-та матем. СО AH CCCP, 1985 , т. 5 , c. 27-44.

28. Sakhanenko A.I. A new way to obtain estimates in the invariance principle. - High Dimensional Probability, II (Seattle, 1999). Boston: Birkhäuser, 2000, p. 223-245. (Progr. Probab., v. 47.)

29. Саханенко А. И. Оценки в принципе инвариантности в терминах срезанных степенных моментов. - Сиб. матем. журн., 2006, т. 47, № 6, с. 1355-1371.

30. Shao Q.-M. Strong approximation theorems for independent random variables and their applications. - J. Multivariate Anal., 1995, v. 52, № 1, p. 107-130.

31. Скороход А.В. Исследования по теории случайных процессов. Киев: Киевский гос. ун-т, 1961, $216 \mathrm{c}$.

32. Зайцев А.Ю. Оценки расстояния Леви-Прохорова в многомерной центральной предельной теореме для случайных векторов с конечными экспоненциальными моментами. - Теория вероятн. и ее примен., 1986, т. 31, в. 2, с. 246-265.

33. Zaitsev A. Yu. Multidimensional version of the results of Komlós, Major and Tusnády for vectors with finite exponential moments. - ESAIM Probab. Statist., 1998, v. 2, p. 41-108.

34. Zaitsev A. Yu. Multidimensional version of the results of Sakhanenko in the invariance principle for vectors with finite exponential moments. I, II, III. - Теория вероятн. и ее примен., 2000 , т. 45 , в. 4 , с. $718-738 ; 2001$, т. 46 , в. 3 , с. $535-561$; в. 4 , с. $744-769$.

35. Zaitsev A. Yu. Estimates for the strong approximation in multidimensional central limit theorem. - Proceedings of the International Congress of Mathematicians (Beijing, 2002). V. III: Invited Lectures. Beijing: Higher Ed. Press, 2002, p. 107-116.

36. Зайчев А. Ю. Оценки точности сильной аппроксимации в многомерном принципе инвариантности. - Записки науч. семин. ПОМИ, 2006, т. 339, с. 37-53.

Поступила в редакцию

31.VII.2007 